\title{
The chicken or the egg: Longitudinal changes in pain and catastrophizing in women with interstitial cystitis/bladder pain syndrome
}

\author{
Alison Crawford, $\mathrm{MSc}^{1}$; Abi Muere, $\mathrm{PhD}^{2}$; Dean A. Tripp, $\mathrm{PhD}^{3}$; J Curtis Nickel, $\mathrm{MD}^{4}$; R. \\ Christopher Doiron, MD, MPH, FRCSC ${ }^{4}$; Robert Moldwin, $\mathrm{MD}^{5}$; Laura Katz $\mathrm{PhD}^{2,6}$ \\ ${ }^{1}$ Department of Psychology, Queen's University, Kingston, ON, Canada; ${ }^{2}$ Michael G. DeGroote Pain Clinic, \\ Hamilton Health Sciences, Hamilton, ON, Canada; ${ }^{3}$ Departments of Psychology, Urology \& Anesthesiology, \\ Queen's University, Kingston, ON, Canada; ${ }^{4}$ Department of Urology, Queen's University, Kingston, ON, Canada; \\ ${ }^{5}$ Zucker School of Medicine at Hofstra-Northwell, New Hyde Park, NY, United States; ${ }^{6}$ Department of Psychiatry \\ and Behavioural Neurosciences, McMaster University, Hamilton, ON, Canada
}

Cite as: Crawford A, Muere A, Tripp DA, et al. The chicken or the egg: Longitudinal changes in pain and catastrophizing in women with interstitial cystitis/bladder pain syndrome. Can Urol Assoc J 2021 March 18; Epub ahead of print. http://dx.doi.org/10.5489/cuaj.7106

Published online March 18, 2021

$* * *$

\section{Abstract}

Introduction: Evidence-based psychological strategies are being used as clinicians look for helpful interventions for patients diagnosed with the enigmatic chronic urological pelvic pain condition of interstitial cystitis/bladder pain syndrome (IC/BPS). Pain and pain catastrophizing are associated with chronic pelvic pain outcomes but the longitudinal role of catastrophizing on patient pain in IC/BPS remains unknown.

Methods: Women with IC/BPS were recruited from tertiary care clinics across North America and completed a battery of questionnaires, including demographics, pain, depression, catastrophizing at baseline, six months, and one year.

Results: A total of 226 patients completed baseline, 183 completed the six-month survey, and 151 completed the one-year survey. Using a cross-lagged analysis, early changes in pain catastrophizing predicted later changes in pain, but not vice versa. Followup subscale analyses revealed that early changes in magnification predicted later changes in pain, early changes in pain predicted later changes in rumination, and that there was a recursive relationship between changes in helplessness and changes in pain across the study.

Conclusions: Pain catastrophizing should be considered a prime target in psychological treatment for chronic pain in patients with IC/BPS, particularly those thinking styles associated with pain onset and maintenance. Future research should be conducted with constructs such as 
pain catastrophizing in samples prioritizing diversity of patients with IC/BPS and mechanisms as to how to effectively decrease catastrophizing.

\section{Introduction}

Interstitial cystitis/bladder pain syndrome (IC/BPS) is a chronic pain condition that is characterized by urinary urgency, frequency, and painful voiding. ${ }^{1}$ This condition affects approximately $2.7 \%$ to $6.5 \%$ of women in the United States, ${ }^{2}$ and $2.8 \%$ of patients in Canadian urology clinics. ${ }^{3}$ There is a lack of consensus on the etiology of IC/BPS which complicates its medical treatment. ${ }^{4}$ Currently, there is no cure for IC/BPS, with many treatments focusing on symptom management and psychosocial interventions. ${ }^{5}$

Pain catastrophizing is considered the most salient, empirically supported psychosocial variable in treatments for IC/BPS for pain. ${ }^{6-8}$ Pain catastrophizing is an anxious cognitive disposition to current or anticipated pain, resulting in greater anxiety about pain and ultimately pain sensitivity. ${ }^{9}$ Models of coping ${ }^{10}$ that are adapted for chronic pain, show that changes in pain catastrophizing predict changes in pain for headache sufferers. ${ }^{11}$ Cross-sectional IC/BPS research also showed that pain catastrophizing at six months was responsible for the relationship between baseline depression and pain at one year. ${ }^{12}$ Finally, other cross-sectional work reports that pain catastrophizing is associated with IC/BPS pain through other psychological coping mechanisms ${ }^{13}$, and for tertiary care IC/BPS cases, pain catastrophizing was associated with greater suicidal ideation. ${ }^{14}$

There are no studies directly investigating the relationship between pain catastrophizing and pain in women with IC/BPS employing a longitudinal design. The present study aimed to examine the directionality of the pain catastrophizing and pain relationship. We hypothesized that (1) earlier changes in pain catastrophizing will predict later changes in pain severity of patients with IC/BPS, and (2) earlier changes in chronic pain levels will not predict later changes in pain catastrophizing. To evaluate whether changes in pain catastrophizing predict future pain and/or changes in pain could predict later changes in pain catastrophizing, a cross-lagged panel analysis was used.

\section{Method}

\section{Participants}

Adult women with a diagnosis of IC/BPS attending tertiary care urology clinics for IC/BPS in Kingston ON, Toronto ON, New York and Tennessee were recruited between 2013 and 2018. Inclusion criteria were self-identifying as a cisgender woman, being able to read and write in English, and being 18 years of age or older. The diagnosis of IC/BPS was established by 
experienced urologists employing the diagnostic criteria from the American Urological Association IC/BPS guideline. ${ }^{5}$

\section{Procedure}

Institutional ethics board clearance was obtained at all sites of study. After their clinic appointment, eligible participants were approached by clinic staff or a research assistant about participating in this study. All potential participants were provided basic information about the study. Individuals who indicated interest in participating were provided a letter of information and consent form pertaining to the study. If consenting, participants then chose to complete questionnaires by paper in the clinic or at home. If they completed the questionnaires at home, they were provided with a questionnaire package that included the measures as well as a stamped envelope for return to the Pain Research Lab at Queen's University. Participants who opted for the online version were emailed a link to the survey. The entire questionnaire package required approximately 35-40 minutes to complete, as estimated based on results from pilot testing.

Participants were asked to complete the same set of questionnaires six months after the initial urology appointment (Time 2) and one-year post-appointment (Time 3). Time 2 and Time 3 questionnaires were sent by mail or electronically based on participant preference. Time points were spaced six months apart in order to capture longer-term changes in variables of interest, consistent with previous cross-lagged research. ${ }^{15,16}$ Five attempts were made to contact participants who did not return their questionnaires. After five unsuccessful attempts, participants were added to the attrition list.

\section{Measures}

Participants were given a battery of questionnaires, including questions regarding demographics, pain, and pain catastrophizing, among other measures.

\section{Demographics}

Demographic questions included age, education, ethnicity, and employment status. Medical history questions included length of IC/BPS diagnosis, co-morbid medical conditions, and the type and frequency of healthcare utilization in the past six months (e.g., number of visits to a pharmacist in the past six months).

Pain

The Short Form - McGill Pain Questionnaire (SF-MPQ) is a self-report measure of current pain quality. ${ }^{17}$ It consists of 15 single-word descriptors of pain; 11 descriptors of sensory pain quality 
(e.g., "stabbing) and four descriptors of affective pain quality (e.g., "punishing-cruel"). Individuals indicate the degree to which the word describes the severity of their pain using a four-point scale ranging from 0 (None) to 3 (Severe). Higher scores indicate more severe pain. ${ }^{18}$

\section{Pain catastrophizing}

The Pain Catastrophizing Scale (PCS) is a measure of an individual's negative appraisal process of a pain experience. ${ }^{9}$ Respondents indicated the degree to which they experienced 13 specified thoughts or feelings (e.g., "I worry all the time whether the pain will end") during a past pain experience on a five-point scale from 0 (Not at all) to 4 (All the time). Three subscale scores can be calculated which assess three interrelated subcomponents of pain catastrophizing: magnification, rumination, and hopelessness. Higher total and subtotal scores indicate greater pain catastrophizing. ${ }^{18}$

\section{Data analysis}

Analyses were conducted using Statistical Packages for the Social Sciences (SPSS, Version 25.0). ${ }^{19}$ Data were inspected for univariate and multivariate normality, outliers, multicollinearity, and missing data. For cases with $80 \%$ or higher completion, missing data were imputed with the average score from the completed items within each measure $(n=30)$. Cases with less than $80 \%$ completion for any measure were removed from analyses involving the total score of the measure of interest $(n=86)$.

Total scores from the SF-MPQ and PCS were calculated for each of the three time points. Residualized change scores between Time 1 and Time 2 (Early Pain Change) were created by regressing Time 1 total scores onto Time 2 total scores for each measure and keeping the residuals. The same procedure was used to create residualized change scores between Time 2 and Time 3 (Later Pain Change). Residualized change scores were used instead of simple change scores to avoid problems of dependence. ${ }^{20}$

The main hypothesis was that earlier changes in catastrophizing predicted later changes in pain but not vice versa (see Figure 1). This was tested using cross-lagged panel analyses that are two hierarchical regressions/models. In Model 1, Later Pain Change was the dependent variable. Early Pain Change and Later Catastrophizing Change were entered first as predictors to control for their predictive effects. Early Catastrophizing Change was entered as a final predictor.

In Model 2, Later Catastrophizing Change was the dependent variable. Early Catastrophizing Change and Later Pain Change were entered first as predictors to control for their predictive effects. Early Pain Change was entered as a final predictor.

See Figure 1 for the conceptual design. The study's main hypothesis will be supported if Early Catastrophizing Change is found to be a significant predictor of Later Pain Change in 
Model 1 and Early Pain Change is not a significant predictor of Later Catastrophizing Change in Model 2.

Follow-up cross-lagged analyses were also run to examine the effects of the subscales of catastrophizing. That is, Models 1 and 2 were re-run with residualized changes in magnification, rumination, and helplessness being examined. This was done to better understand exactly how the subcomponents that constitute pain catastrophizing function in relation to pain over time.

\section{Results}

Approximately 380 IC/BPS patients were invited to participate in the study. Data from 226 participants were collected (Time 1 [baseline] $n=226-62$ opted for online version; Time 2 [six months post-baseline] $n=183$; Time 3 [one-year post-baseline] $n=151$ ). Regarding participants with missing data who were removed from study analyses $(\mathrm{n}=86), 75$ participants were missing multiple questionnaires at Time 2 and/or Time 3 and were added to the attrition list. A total of 11 participants were missing a single (random) questionnaire from Time 1, 2, or 3 .

See Table 1 for details on demographic characteristics of the sample at Time 1, Time 2, and Time 3. Cronbach's alpha for all items of the SF-MPQ was acceptable at each time point (Time $1=.919$, Time $2=.934$, Time $3=.934$ ). Cronbach's alpha for all items of the PCS was acceptable at each time point (Time $1=.957$, Time $2=.963$, Time $3=.959)$.

\section{Primary analyses}

As shown in Table 2, increases in Later Catastrophizing Change predicted increases in Later Pain Change and an increase in Early Pain Change predicted a decrease in Later Pain Change. Increases in Early Catastrophizing Change predicted increases in Later Pain Change, after controlling for the variables entered in the first step.

As shown in Table 3, increases in Later Pain Change predicted increases in Later Catastrophizing Change. However, Early Catastrophizing Change did not significantly predict Later Catastrophizing Change. Early Pain Change did not significantly predict Later Catastrophizing Change, after controlling for the variables entered in the first step. Taken together, results from Models 1 and 2 supported the hypothesis that earlier changes in catastrophizing predict later changes in pain but not vice versa.

\section{Followup analyses}

As stated in the data analyses section, follow-up analyses were run to explore if cross-lagged panel analyses using the subscales of catastrophizing would produce similar results to catastrophizing as a whole. For brevity, the most important findings will be highlighted. Please see the supplementary table for a comprehensive view of all findings. The most notable findings include: early changes in magnification predicted later changes in pain $(b=1.15, t(139)=3.90, p$ $<.001)$, early changes in pain predicted later changes in rumination $(b=0.07, t(139)=2.26, p=$ $.03)$, early changes in pain predicted later changes in helplessness $(b=0.09, t(139)=2.23, p=$ 
$.03)$, and early changes in helplessness predicted later changes in pain $(b=0.50, t(139)=3.39, p$ $=.001)$.

\section{Discussion}

This study aimed to test the directionality of the catastrophizing-pain relationship in a sample of women with IC/BPS. The cross-lagged panel analyses demonstrate that earlier changes in pain catastrophizing predict later changes in pain. In contrast, earlier changes in pain did not predict later changes in pain catastrophizing. Taken together, results suggest that increases in catastrophizing precede increases in pain, but not vice versa, over the course of a 12-month period of IC/BPS pain.

These findings are consistent with previous research testing the directionality of the catastrophizing-pain relationship using cross-lagged panel analyses in both patients with chronic pain and pain-free individuals. ${ }^{15,21}$ Although the current study used a non-experimental longitudinal design, the results were comparable to experimental research that induced both catastrophizing and acute pain among pain-free individuals. ${ }^{21}$ Therefore, we can more confidently conclude that catastrophizing has a predictive effect on IC/BPS pain in women.

It is important to note that the follow-up analyses of the pain catastrophizing subscales (i.e., rumination, magnification, helplessness) produced inconsistent results. For example, early changes in magnification predicted later changes in pain, but not vice versa. However, the reverse relationship was found with rumination; earlier changes in pain predicted later changes in rumination but not vice versa. Early increases in helplessness over managing pain predicted later increases in pain levels, and also earlier increases in pain levels predicted later increases in helplessness. This pattern of results suggests that the helplessness-pain relationship may be more recursive or bidirectional (i.e., creating a vicious self-perpetuating cycle) in IC/BPS, and that the rumination and magnification may have differing temporal relationships to IC/BPS pain.

In summary, these results indicate that changes in pain catastrophizing predict future pain, and that helplessness may be a key feature of this relationship. Although both magnification and rumination play a role in the maintaining the relation between catastrophizing and pain, it appears that helplessness is a potential mechanism that perpetuates the catastrophizing-pain relationship over time. Thus, patients presenting with elevated pain or pain catastrophizing may benefit from referral to mental health support. ${ }^{22}$ Cognitive-Behavioural Therapy (CBT) and Acceptance and Commitment Therapy (ACT) are two intervention orientations found to decrease pain catastrophizing in patients with chronic pain. ${ }^{23-25} \mathrm{CBT}$ orientations emphasize challenging the content of thoughts (like catastrophizing) whereas ACT orientations aim to challenge the utility of certain thoughts (like catastrophizing) through acceptance. ${ }^{26}$ Both of these orientations focus on promoting more adaptive emotion regulation strategies while unlearning strategies that are no longer helpful. It is important to note that catastrophizing was once adaptive for pain and emotion regulation, in the case of many patients. 
Some theories posit that catastrophizing is an avoidant coping strategy that reduces the intensity of fear and worry that patients have about their pain. ${ }^{27}$ Although pain catastrophizing may have been helpful for patients coping with the unknowns of their symptoms in the past, both CBT and ACT can help patients find ways to cope more effectively with their pain and fear presently and in the future.

This longitudinal study has some limitations. The overwhelming majority of participants in the present study were White (95.1-98.0\% across the three time points), whereas estimates from a large-scale, community-based epidemiological survey in the United States found that approximately $25.4 \%$ of women with IC/BPS self-identified as being a racial minority. ${ }^{28}$ Participants were recruited from a tertiary care setting and there was no reimbursement offered for participation. Thus, it is possible that there was a self-selection bias in the study sample, both between initially recruited participants and the general IC/BPS population, as well as between initially recruited participants and the final study sample.

Additionally, approximately one third of Time 1 participants were lost to attrition by Time 3. Attrition analyses indicated that participants who completed all three questionnaire packages were significantly older, had lower pain levels, and lower levels of depression symptoms than participants lost to follow-up after completing the first questionnaire package (i.e., Time 1). Participants lost to attrition did not differ significantly with regard to IC/BPS symptomology severity and pain catastrophizing levels. Moreover, while attrition analyses do indicate that participants who completed all three time points did have significantly lower pain scores in comparison to individuals who only completed questionnaires at Time 1, the study's main analyses (cross-lagged analyses) only included participants who completed the variables of interest (the PCS and the SF-MPQ) at all three time points. Therefore, the residual zed change scores used in the cross-lagged analyses are not affected by attrition effects.

Future studies must make concerted efforts to recruit more ethnically representative samples of the IC/BPS population. Although not excusable, limited diversity in health research is not uncommon. Thankfully, researchers have recently tested various effective methods for increasing diversity in health research samples, such as creating and using a community research registry. ${ }^{29,}{ }^{30}$ Additionally, although the majority of individuals with IC/BPS are women, ${ }^{3}$ diversity in health research should make efforts to include more men. Moreover, future research should examine mechanisms for effective de-catastrophizing in this population. At the same time, as only $19 \%$ of the variation in later changes in pain are accounted for by earlier changes in pain, earlier changes in catastrophizing, and later changes in catastrophizing, future research should consider other patient and disease characteristics (e.g., severity of IC/BPS symptomology).

As a final thought, the term pain catastrophizing may be perceived as pejorative to some patients and advocates, and some recent discussion in the broader academic and clinical community have sought to reconceptualize this term as pain-related worry (or pain distress or 
pain-related fear). However, pain catastrophizing is a predictive and significant clinical process variable with a strong theoretical research presence spanning a number of decades (e.g., over 8,000 citations). To engage in meaningful attempts to change such terminology, building an evidence base that would show the newly named construct to be linked, with respect to range and magnitude, to the host of variables that have been shown to be associated with pain catastrophizing is suggested. Further, the future research efforts for any measure used to assess the alternate construct should demonstrate predictive power superior to that of the pain catastrophizing scale.

\section{Conclusions}

Pain catastrophizing is again confirmed to be an important psychosocial process variable in the pain experience of IC/BPS. This study demonstrated that changes in pain catastrophizing predicts later changes in pain in IC/BPS and should therefore be a therapeutic focus for the management of future pain levels. To help validate patient experiences, urologists can explain to patients that both their biology and thinking patterns work in tandem to influence their real experience of pain. Patients benefit from thinking about pain as a stressor, because many patients are aware of how social or psychological stress can impact their bodies and how their bodily symptoms can create psychological stress. Although urologists are well equipped to support with the biological side of their illness, a psychologist or mental health practitioner with experience in pain can be another important piece of patient symptom management. More research is required to clarify the exact role that the subcomponents of pain catastrophizing play in the patient pain experience in a more diverse IC/BPS population. 


\section{References}

1. Nickel JC, Shoskes D, Irvine-Bird K. Clinical phenotyping of women with interstitial cystitis/painful bladder syndrome: a key to classification and potentially improved management. J Urol 2009; 182: 155-60.

2. Berry SH, Elliott MN, Suttorp M, et al. Prevalence of symptoms of bladder pain syndrome/interstitial cystitis among adult females in the United States. J Urol 2011; 186: $540-44$.

3. Nickel JC, Teichman JM, Gregoire M, et al. Prevalence, diagnosis, characterization, and treatment of prostatitis, interstitial cystitis, and epididymitis in outpatient urological practice: the Canadian PIE Study. Urology 2005; 66: 935-40.

4. Fang $\mathrm{ZW}, \mathrm{Xu} \mathrm{KX}$. Interstitial cystitis/bladder pain syndrome: a review and an update. Curr Bladder Dysfunct Rep 2016; 11: 391-98.

5. Hanno PM, Erickson D, Moldwin R, et al. Diagnosis and treatment of interstitial cystitis/bladder pain syndrome: AUA guideline amendment. J Urol 2015; 193: 1545-53.

6. Sewell M, Churilov L, Mooney S, et al. Chronic pelvic pain - pain catastrophizing, pelvic pain and quality of life. Scand J Pain 2018; 18: 441-48.

7. Chen A, Argoff C, Crosby E, et al. Chronic pelvic pain patients demonstrate higher catastrophizing in association with pelvic symptoms and comorbid pain diagnoses. Urology 2020.

8. Huang X, Qin Z, Cui H, et al. Psychological factors and pain catastrophizing in men with chronic prostatitis/chronic pelvic pain syndrome (CP/CPPS): a meta-analysis. Transl Androl Urol 2020; 9: 485-93.

9. Sullivan MJ, Bishop SR, Pivik J. The Pain Catastrophizing Scale: development and validation. Psychol Assess 1995; 7: 524-32.

10. Lazarus RS, Folkman S. Stress, Appraisal, and Coping. New York, NY: Springer Pub. Co. 1984.

11. Kjogx H, Kasch H, Zachariae R, et al. Experimental manipulations of pain catastrophizing influence pain levels in patients with chronic pain and healthy volunteers. Pain 2016; 157: 1287-96.

12. Crawford A, Tripp DA, Nickel JC, et al. Depression and helplessness impact interstitial cystitis/bladder pain syndrome pain over time. Can Urol Assoc J 2019; 13: 328-33.

13. Muere A, Tripp DA, Nickel JC, et al. Depression and coping behaviors are key factors in understanding pain in interstitial cystitis/bladder pain syndrome. Pain Manag Nurs 2018.

14. Tripp D, Nickel JC, Krsmanovic A, et al. Depression and catastrophizing predicts suicidal ideation in tertiary patients with interstitial cystitis/bladder pain syndrome (IC/BPS). J Urol 2016; 195: e956.

15. Campbell CM, McCauley L, Bounds SC, et al. Changes in pain catastrophizing predit later changes in fibromyalgia clincal and experimental pain report: cross-lagged panel analyses of dispositional and situational catastrophizing. Arthritis Res.Ther 2012; 14: R231. 
16. Lerman SF, Rudich Z, Brill S, et al. Longitudinal associations between depression, anxiety, pain, and pain-related disability in chronic pain patients. Psychosom Med 2015; 77: 333-41.

17. Melzack R. The short-form McGill Pain Questionnaire. Pain 1987; 30: 191-97.

18. Kline P. The handbook of psychological testing. 2nd ed. London, UK: Routledge. 1999.

19. Corp. I, IBM SPSS Statistics for Macintosh. 2017, IBM Corp.: Armonk, NY.

20. Cohen P, Cohen J, West SG, et al. Applied multiple regression/correlation analysis for the behavioral sciences. 3rd ed. Mahwah, NJ: Lawrence Erlbaum Associates, Inc. 2001.

21. Campbell CM, Kronfli T, Buenaver LF, et al. Situational versus dispositional measurement of catastrophizing: associations with pain responses in multiple samples. $J$ Pain 2010; 11: 443-53 e2.

22. Tripp DA, Nickel JC. The psychology of urologic chronic pelvic pain: A primer for urologists who want to know how to better manage chronic prostatitis and interstitial cystitis. AUA Update Ser 2011; 30: 385-96.

23. Kerns RD, Sellinger J, Goodin BR. Psychological treatment of chronic pain. Annu Rev Clin 2011; 7.

24. McCracken LM, Vowles KE. Acceptance and commitment therapy and mindfulness for chronic pain. Am Psychol 2014; 69: 178-87.

25. Wetherell JL, Afari N, Rutledge T, et al. A randomized, controlled trial of acceptance and commitment therapy and cognitive-behavioral therapy for chronic pain. Pain 2011; 152: 2098-107.

26. Hofmann SG, Asumndson GJG. Acceptance and mindfulness-based therapy: New wave or old hat? Clin Psychol Rev 2008; 28: 1-16.

27. Flink IL, Boersma K, Linton SJ. Pain catastrophizing as repetitive negative thinking: a development of the conceptualization. Cogn Beahv Ther 2013; 42: 215-23.

28. Konkle KS, Berry SH, Elliott MN, et al. Comparison of an Interstitial Cystitis / Bladder Pain Syndrome clinical cohort with symptomatic community women from the RAND Interstitial Cystitis Epidemiology Study. J Urol 2012; 187: 508-12.

29. Amorrortu RPS, Arevalo M, Vernon SW, et al. Recruitment of racial and ethinic minorities to clinical trials conducted within specialty clinics: an intervention mapping approach. Trials 2018; 19.

30. Bishop WP, Tiro JA, Sanders JM, et al. Effectiveness of a community research registry to recruit minority and underserved adults for health research. Clin Transl Sci 2015; 8: 8284. 
Fig. 1. Cross-lagged panel analyses. Green arrows indicate predicted significant effects. Cat: catastrophizing.

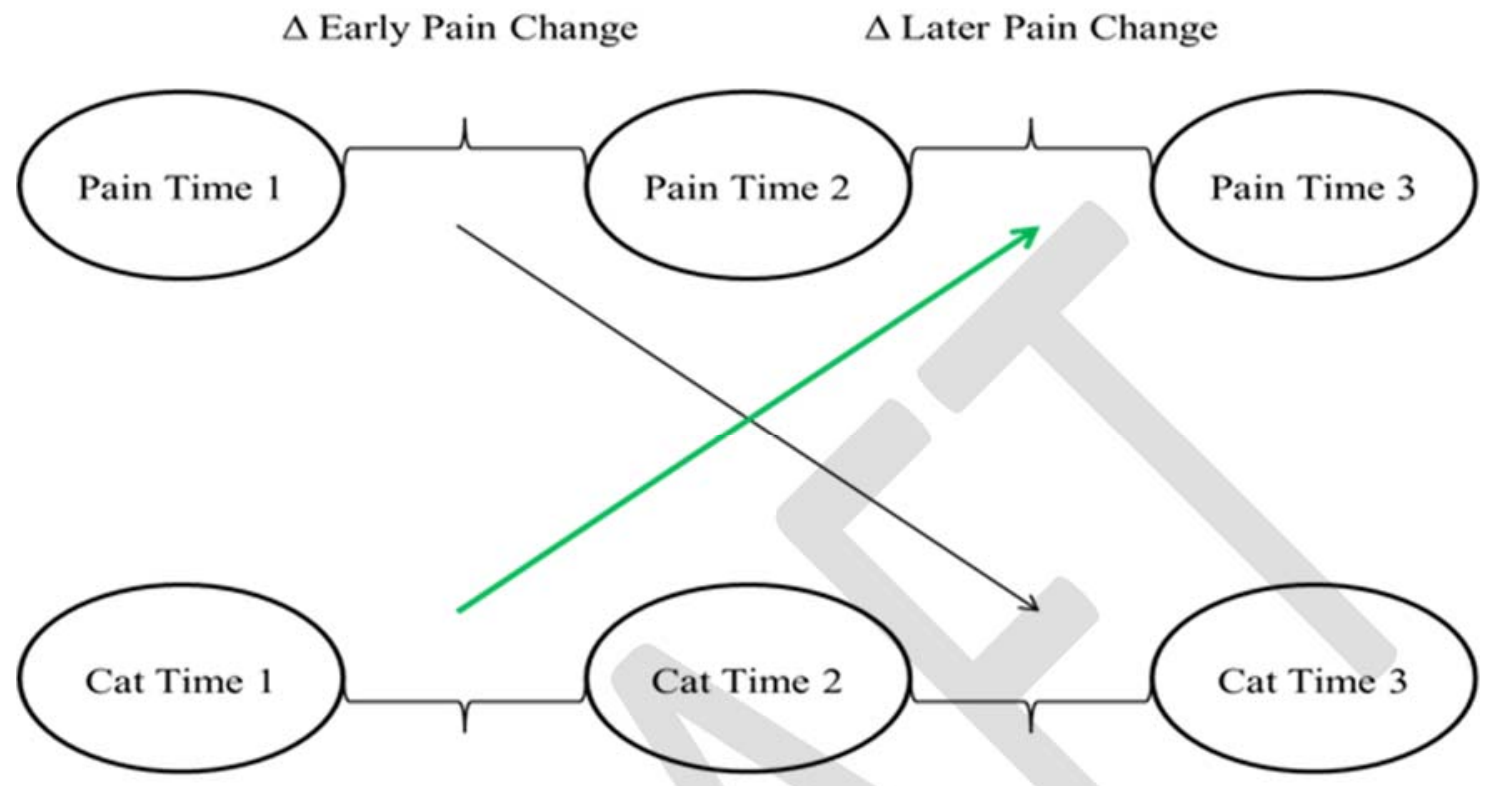

$\Delta$ Early Cat Change

$\Delta$ Later Cat Change

\begin{tabular}{|c|c|c|c|}
\hline & $\begin{array}{l}\text { Time } 1 \\
(\mathrm{n}=226)\end{array}$ & $\begin{array}{c}\text { Time } 2 \\
(n=183)\end{array}$ & $\begin{array}{l}\text { Time } 3 \\
(n=151)\end{array}$ \\
\hline \multicolumn{4}{|l|}{ Age (years) } \\
\hline Mean \pm SD & $49.29 \pm 15.67$ & $51.53 \pm 15.47$ & $53.22 \pm 14.82$ \\
\hline Range & $18-85$ & $20-85$ & $21-86$ \\
\hline Time since diagnosis (mean years $\pm \mathrm{SD}$ ) & $7.95 \pm 8.23$ & $8.91 \pm 8.54$ & $9.84 \pm 8.44$ \\
\hline \multicolumn{4}{|l|}{ Ethnicity, n (\%) } \\
\hline White & $215(95.1 \%)$ & $176(96.7 \%)$ & $148(98.0 \%)$ \\
\hline Other & $11(4.9 \%)$ & $6(3.2 \%)$ & $3(2.0 \%)$ \\
\hline \multicolumn{4}{|l|}{ Employment, n (\%) } \\
\hline Employed & $99(43.8 \%)$ & $80(43.7 \%)$ & $62(41.1 \%)$ \\
\hline $\begin{array}{l}\text { Not employed (unemployed, retired, } \\
\text { disabled, student) }\end{array}$ & $125(55.3 \%)$ & $103(56.3 \%)$ & $89(58.9 \%)$ \\
\hline Married, $\mathrm{n}(\%)$ & $143(63.3 \%)$ & $113(62.4 \%)$ & $95(63.3 \%)$ \\
\hline \multicolumn{4}{|l|}{ Location, $\mathrm{n}(\%)$} \\
\hline Kingston & $140(61.9 \%)$ & $114(62.3 \%)$ & 90 \\
\hline Toronto & $28(12.4 \%)$ & $27(14.8 \%)$ & $27(17.9 \%)$ \\
\hline
\end{tabular}




\begin{tabular}{|l|c|c|c|}
\hline New York & $57(25.2 \%)$ & $40(21.9 \%)$ & $33(21.9 \%)$ \\
\hline Tennessee & $1(0.4 \%)$ & 0 & 0 \\
\hline Not provided & 0 & $2(1.1 \%)$ & $1(0.7 \%)$ \\
\hline SF-MPQ score (mean \pm standard deviation) & $16.77 \pm 11.19$ & $14.27 \pm 11.32$ & $13.11 \pm 10.98$ \\
\hline PCS score (mean \pm standard deviation) & $23.68 \pm 14.39$ & $19.88 \pm 14.36$ & $17.96 \pm 3.05$ \\
\hline Rumination & $8.33 \pm 5.09$ & $6.97 \pm 5.22$ & $6.25 \pm 4.74$ \\
\hline Magnification & $4.48 \pm 3.41$ & $3.84 \pm 3.35$ & $3.40 \pm 2.98$ \\
\hline Helplessness & $10.87 \pm 6.96$ & $9.07 \pm 6.70$ & $8.31 \pm 6.19$ \\
\hline Comorbid diagnoses & & & \\
\hline Fibromyalgia & $52(23.0 \%)$ & $43(23.5 \%)$ & $37(24.5 \%)$ \\
\hline Chronic fatigue syndrome & $24(10.6 \%)$ & $25(13.7 \%)$ & $21(13.9 \%)$ \\
\hline Urinary tract infections & $148(65.5 \%)$ & $117(63.9 \%)$ & $88(58.3 \%)$ \\
\hline Inflammatory bowel disease & $18(8.0 \%)$ & $19(10.4 \%)$ & $19(12.6 \%)$ \\
\hline Irritable bowel syndrome & $99(43.8 \%)$ & $79(43.2 \%)$ & $75(49.7 \%)$ \\
\hline Tension headaches & $46(20.4 \%)$ & $30(16.4 \%)$ & $24(15.9 \%)$ \\
\hline Migraines & $63(27.9 \%)$ & $48(26.2 \%)$ & $46(30.5 \%)$ \\
\hline Low back pain & $103(45.6 \%)$ & $78(42.6 \%)$ & $62(41.1 \%)$ \\
\hline Vulvodynia & $44(19.5 \%)$ & $38(20.8 \%)$ & $30(19.9 \%)$ \\
\hline Temporomandibular joint disorder & $33(14.6 \%)$ & $33(18.0 \%)$ & $29(19.2 \%)$ \\
\hline $\begin{array}{l}\text { Other (various concerns, e.g., arthritis, } \\
\text { depression, endometriosis, etc.) }\end{array}$ & $64(28.3 \%)$ & $46(25.1 \%)$ & $40(26.5 \%)$ \\
\hline
\end{tabular}

PCS: pain catastrophizing scale; SD: standard deviation; SF-MPQ: Short-form McGill Pain Questionnaire. 


\begin{tabular}{|c|c|c|c|c|c|c|c|}
\hline \multicolumn{7}{|c|}{ Table 2. Hierarchical regression analysis predicting later pain change in Model 1 } \\
\hline & $\mathrm{b}$ & $\mathrm{SE}$ & $\beta$ & $\mathrm{t}$ & $\mathrm{F}$ change & $\begin{array}{c}\mathrm{R}^{2} \\
\text { change }\end{array}$ & $\mathrm{R}^{2}$ \\
\hline Step 1: & & & & & $11.62^{* * *}$ & 0.14 & 0.14 \\
\hline Early pain change & -0.16 & 0.08 & -0.16 & $-2.05^{*}$ & & & \\
\hline Later cat change & 0.37 & 0.08 & 0.35 & $4.42^{* * *}$ & & & \\
\hline Step 2: & & & & & $8.62^{* *}$ & 0.05 & 0.19 \\
\hline Early pain change & -0.24 & 0.08 & -0.24 & $-2.98^{* *}$ & & & \\
\hline Later cat change & 0.38 & 0.08 & 0.36 & $4.72^{* * *}$ & & & \\
\hline Early cat change & 0.21 & 0.07 & 0.24 & $2.94^{* *}$ & & & \\
\hline
\end{tabular}

Cat: catastrophizing. ${ }^{*} \mathrm{p}<0.05 ;{ }^{* *} \mathrm{p}<0.01 ;{ }^{* * *} \mathrm{p}<0.001$.

\begin{tabular}{l} 
Table 3. Hierarchical regression analysis predicting later catastrophizing change in \\
Model 2 \\
\hline
\end{tabular}

Cat: catastrophizing. ${ }^{*} \mathrm{p}<0.001$. 


\begin{tabular}{|c|c|c|c|c|c|c|c|}
\hline \multicolumn{8}{|c|}{ Supplementary Table 1. Followup complete results } \\
\hline \multicolumn{8}{|c|}{ Hierarchical regression analysis predicting later pain change } \\
\hline & $\mathrm{b}$ & SE & $\beta$ & $\mathrm{t}$ & F change & $\begin{array}{c}\mathrm{R}^{2} \\
\text { change }\end{array}$ & $\mathrm{R}^{2}$ \\
\hline Step 1: & & & & & $5.76^{* *}$ & 0.08 & 0.08 \\
\hline Early pain change & -0.14 & 0.08 & -0.14 & -1.72 & & & \\
\hline Later mag change & 1.00 & 0.35 & 0.23 & $2.84^{* *}$ & & & \\
\hline Step 2: & & & & & $15.17^{* * *}$ & 0.09 & 0.17 \\
\hline Early pain change & -0.24 & 0.08 & -0.25 & $0.003^{* *}$ & & & \\
\hline Later mag change & 1.06 & 0.34 & 0.25 & $3.16^{* *}$ & & & \\
\hline Early mag change & 1.15 & 0.30 & 0.32 & $3.90^{* * *}$ & & & \\
\hline \multicolumn{8}{|c|}{ Hierarchical regression analysis predicting later magnification change } \\
\hline Step 1: & & & & & $5.27^{* *}$ & 0.07 & 0.07 \\
\hline Early mag change & -0.10 & 0.07 & -0.12 & -1.43 & & 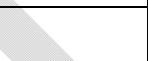 & \\
\hline Later pain change & 0.06 & 0.02 & 0.26 & $3.16^{* *}$ & & 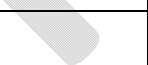 & \\
\hline Step 2: & & & & +2 & 0.18 & 0.001 & 0.07 \\
\hline Early mag change & -0.11 & 0.08 & -0.13 & -1.48 & & & \\
\hline Later pain change & 0.06 & 0.02 & 0.27 & $3.16^{* *}$ & 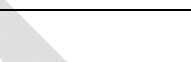 & & \\
\hline Early pain change & 0.01 & 0.02 & 0.04 & 0.43 & + & & \\
\hline \multicolumn{8}{|c|}{ Hierarchical regression analysis predicting later pain change } \\
\hline Step 1: & & & +1 & & $13.90^{* * * *}$ & 0.17 & 0.17 \\
\hline Early pain change & -0.18 & 0.08 & -0.19 & $-2.38^{*}$ & & & \\
\hline $\begin{array}{l}\text { Later rumination } \\
\text { change }\end{array}$ & 0.99 & 0.20 & 0.38 & $4.90^{* * *}$ & & & \\
\hline Step 2: & 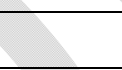 & . & & & 2.28 & 0.01 & 0.18 \\
\hline Early pain change & -0.21 & 0.08 & -0.22 & $-2.70^{* *}$ & & & \\
\hline $\begin{array}{l}\text { Later rumination } \\
\text { change }\end{array}$ & 1.02 & 0.20 & 0.39 & $5.05^{* * *}$ & & & \\
\hline $\begin{array}{l}\text { Early rumination } \\
\text { change }\end{array}$ & 0.26 & 0.17 & 0.12 & 1.51 & & & \\
\hline \multicolumn{8}{|c|}{ Hierarchical regression analysis predicting later rumination change } \\
\hline Step 1: & & & & & $11.51^{* * *}$ & 0.14 & 0.14 \\
\hline $\begin{array}{l}\text { Early rumination } \\
\text { change }\end{array}$ & -0.08 & 0.07 & -0.10 & -1.24 & & & \\
\hline Later pain change & 0.14 & 0.03 & 0.37 & $4.68^{* * *}$ & & & \\
\hline Step 2: & & & & & $5.10^{*}$ & 0.03 & 0.17 \\
\hline
\end{tabular}




\begin{tabular}{|c|c|c|c|c|c|c|c|}
\hline $\begin{array}{l}\text { Early rumination } \\
\text { change }\end{array}$ & -0.12 & 0.07 & -0.14 & -1.78 & & & \\
\hline Later pain change & 0.15 & 0.03 & 0.40 & $5.05^{* * *}$ & & & \\
\hline Early pain change & 0.07 & 0.03 & 0.18 & $2.26^{*}$ & & & \\
\hline \multicolumn{8}{|c|}{ Hierarchical regression predicting later pain change } \\
\hline Step 1: & & & & & $8.68^{* * *}$ & 0.11 & 0.11 \\
\hline Early pain change & -0.16 & 0.08 & -0.17 & $-2.10^{*}$ & & & \\
\hline $\begin{array}{l}\text { Later helpless } \\
\text { change }\end{array}$ & 0.64 & 0.17 & 0.30 & $3.71^{* * *}$ & & & \\
\hline Step 2: & & & & 2 & $11.47^{* *}$ & 0.07 & 0.18 \\
\hline Early pain change & -0.27 & 0.08 & -0.27 & $-3.28^{* *}$ & & & \\
\hline $\begin{array}{l}\text { Later helpless } \\
\text { change }\end{array}$ & 0.72 & 0.17 & 0.33 & $4.29^{* * *}$ & & & \\
\hline $\begin{array}{l}\text { Early helpless } \\
\text { change }\end{array}$ & 0.50 & 0.15 & 0.28 & $3.39^{* *}$ & & & \\
\hline \multicolumn{8}{|c|}{ Hierarchical regression predicting later helplessness change } \\
\hline Step 1: & & & +2 & +2 & $8.68^{* * *}$ & 0.11 & 0.11 \\
\hline Early pain change & -0.16 & 0.08 & -0.17 & $-2.10^{*}$ & & & \\
\hline $\begin{array}{l}\text { Later helpless } \\
\text { change }\end{array}$ & 0.64 & 0.17 & 0.30 & $3.71^{* * *}$ & s & & \\
\hline Step 2: & & & 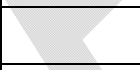 & & $11.47^{* *}$ & 0.07 & 0.18 \\
\hline Early pain change & -0.27 & 0.08 & -0.27 & $-3.28^{* *}$ & & & \\
\hline $\begin{array}{l}\text { Later helpless } \\
\text { change }\end{array}$ & 0.72 & 0.17 & 0.33 & $4.29^{* * *}$ & & & \\
\hline $\begin{array}{l}\text { Early helpless } \\
\text { change }\end{array}$ & 0.50 & 0.15 & 0.28 & $3.39^{* *}$ & & & \\
\hline
\end{tabular}

${ }^{*} \mathrm{p}<0.05 ;{ }^{* *} \mathrm{p}<0.01 ;{ }^{* * *} \mathrm{p}<0.001$. Mag: magnification; helpless: helplessness, 